\title{
Conversion of Fructose into Levulinic Acid by Catalysis of Ionic Liquid
}

\author{
L. LIU* \\ Department of Chemistry \\ Dalian University of Technology \\ Dalian, China \\ e-mail: lliu@dlut.edu.cn
}

\author{
H. REN \\ Department of Chemistry \\ Dalian University of Technology \\ Dalian, China \\ e-mail: lliu@dlut.edu.cn
}

\begin{abstract}
Yield of levulinic acid was obtained from fructose conversion catalyzed by acidic ionic liquid in water. The chemical structures and morphologies of humin byproduct were investigated, indicating three stages of transition for the first time. At the early stage, 5-hydroxymethylfurfural (HMF) intermediate from fructose dehydration can take part in humin formation through two sites. When HMF intermediate was completely converted, humins can react further on aldehyde groups, as evidenced by disappearance of 2 -formylfuran rings. If the reaction time was long enough, humins can undergo subsequent aromatization, with the furan rings reduced and the $\mathrm{C}=\mathrm{C}$ groups increased.
\end{abstract}

Keywords-fructose; levulinic acid; ionic liquid; biomass

\section{INTRODUCTION}

Due to diminishing fossil resources and increasing concern about sustainable development, biomass conversion to fuels and chemicals has attracted a lot of interests [1]. As one of the identified platform chemicals, levulinic acid (LA) serves as a versatile building block for fuel additives, polymer precursors, herbicides, pharmaceutical, flavour substances and chemical intermediates [2]. Converting monosaccharide biomass of fructose to LA could be regarded as a model at molecular level to understand the reaction mechanism easily. Recently, $\mathrm{InCl}_{3}$ was used to catalyze the conversion of fructose into LA with a yield of $45 \%$ in water [3]. Meanwhile, conversion of fructose to LA catalyzed by $\mathrm{H}_{2} \mathrm{SO}_{4}$ in $\gamma$ valerolactone was reported, leading to ca. $70 \%$ yield of LA [4]. During the process of fructose conversion, dark-brown insoluble byproducts, known as humins, are unavoidably formed. Most of the researches in biomass conversion have been focused on the reaction pathway of soluble products such as 5-hydroxymethylfurfural (HMF) and LA, whereas little attention has been paid to the formation and structure of humins. However humins oftentimes compete with desired products, restrain the catalytic activity, and hinder the catalyst reuse and product separation. Thereby elucidation of humins mechanism and inhibition of humins formation will help to improve the efficiency of biomass conversion process.

Several groups have made efforts to understand the mechanism and chemical structure of humins from fructose conversion. For hydrothermal treatment of fructose in the absence of any additional catalysts at $120-140{ }^{\circ} \mathrm{C}$, Yao et al. [5] proposed that fructose first undergoes intramolecular dehydration to HMF, and HMF further polymerizes to form carbon spheres, which contain a dense hydrophobic carbon core and a hydrophilic shell according to field emission scanning electron microscopy (FE-SEM) and transmission electron microscopy (TEM) images. For $\mathrm{H}_{2} \mathrm{SO}_{4}$-catalyzed fructose conversion at $135{ }^{\circ} \mathrm{C}$, Patil et al. [6] suggested that fructose must first be converted to 2,5-dioxo-6hydroxyhexanal (DHH), which subsequently undergoes aldol addition and condensation to form humins. For hexose derived humins catalyzed by $\mathrm{H}_{2} \mathrm{SO}_{4}$ at higher temperature of $180{ }^{\circ} \mathrm{C}$, van Zandvoort et al. [7] inferred a polyfuranic molecular structure, wherein the inclusion of $\mathrm{DHH}$ and limited LA occurs through aldol condensations with the aldehyde groups of HMF or DHH itself.

In our previous work, it was found that $\mathrm{SO}_{3} \mathrm{H}-$ functionalized acidic ionic liquids especially 1-methyl-3-(3sulfopropyl)imidazolium hydrogen sulfate $\left(\left[\mathrm{C}_{3} \mathrm{SO}_{3} \mathrm{Hmim}\right]\right.$ $\mathrm{HSO}_{4}$ ), are environmentally friendly catalysts to convert cellulose into LA with high yield and selectivity [8,9]. Herein, we report on the acidic ionic liquid-catalyzed fructose conversion to platform chemical LA in a yield up to $76 \%$ (Scheme 1), whereas chemical structure and morphology of humin byproducts were investigated under the same reaction conditions.

\section{EXPERIMENTAL SECTION}

D-Fructose (99\%) was purchased from Beijing Solarbio Science \& Technology Co., Ltd. The ionic liquid 1-methyl-3(3-sulfopropyl)imidazolium hydrogen sulfate $\left(\left[\mathrm{C}_{3} \mathrm{SO}_{3} \mathrm{Hmim}\right]\right.$ $\mathrm{HSO}_{4}$ ) was synthesized by acidification of the zwitterion according to literature [8] and characterized by NMR before use. ${ }^{1} \mathrm{H}$ NMR (400 MHz, $\left.\mathrm{D}_{2} \mathrm{O}\right): \delta=8.26(\mathrm{~s}, 1 \mathrm{H} ; \mathrm{N}-\mathrm{CH}-\mathrm{N})$, 7.05 (s, 1H; CH), 6.99 (s, 1H; CH), 3.89 (t, $J=7$ Hz, 2H; N$\mathrm{CH}_{2}$ ), 3.43 (s, 3H; N-CH ${ }_{3}$ ), 2.46 (t, $J=7 \mathrm{~Hz}, 2 \mathrm{H} ; \mathrm{CH}_{2}-\mathrm{S}$ ), 1.80-1.88 ppm (m, 2H; $\left.\mathrm{CH}_{2}\right) ;{ }^{13} \mathrm{C}$ NMR (100 MHz, $\left.\mathrm{D}_{2} \mathrm{O}\right)$ : $\delta=135.6,123.2,121.7,47.2,46.7,35.2,24.5 \mathrm{ppm}$.

The NMR spectra were recorded on Varian INOVA 400 $\mathrm{MHz}$ spectrometer. The infrared spectra were recorded on Bruker Equinox 55 infrared spectrometer. Elemental analyses were determined with the instrument Elementar Vario EL III CHN analyzer. Scanning electron microscopy (SEM) images were acquired on Nova NanoSEM 450 field emotion scanning electron microscopy, whereby samples were coated with a thin Aurum film. 


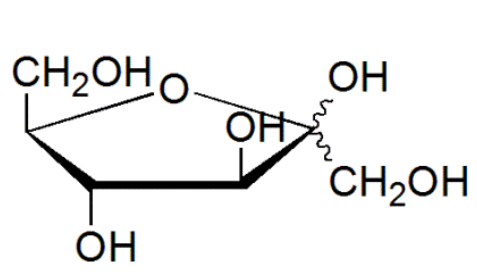

fructose

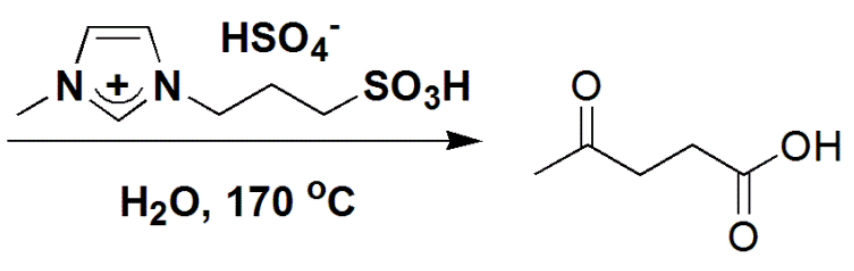

levulinic acid

Scheme 1. Ionic liquid-catalyzed fructose conversion to levulinic acid (LA)

Fructose $(0.925 \mathrm{mmol}), \quad\left[\mathrm{C}_{3} \mathrm{SO}_{3} \mathrm{Hmim}\right] \mathrm{HSO}_{4} \quad(1.000 \mathrm{~g})$ and de-ionized water $(6.000 \mathrm{~g})$ were mixed in a $30 \mathrm{~mL}$ autoclave with Teflon lining and heated in an oil bath at $170^{\circ} \mathrm{C}$ for $0,12,20,30,40,50,60,80 \mathrm{~min}, 3 \mathrm{~h}$, and $12 \mathrm{~h}$. After reaction, the autoclave was cooled in an ice bath immediately. The crude product was analyzed by ${ }^{1} \mathrm{H}$ NMR using ionic liquid as internal standard. The humin solid was filtered, washed with $15 \mathrm{~mL}$ of water for three times, dried at $80{ }^{\circ} \mathrm{C}$ for at least $24 \mathrm{~h}$ and sent to instrumental analysis.

\section{RESULTS AND DISCUSSION}

During the fructose conversion to LA, HMF was observed as intermediate from intramolecular dehydration of fructose. As shown in Fig. 1, the yield of HMF rose up to $21 \%$ with the reaction time increasing from 12 to $20 \mathrm{~min}$, and decreased afterwards, whereas no HMF could be detected in liquid phase after reaction for $50 \mathrm{~min}$. On the other hand, the yield of LA ascended to $71.0 \%$ at $50 \mathrm{~min}$, indicating HMF intermediate underwent rehydration to form LA. The yield of LA increased slightly to $76.3 \%$ after reaction for $3 \mathrm{~h}$ and leveled off, for $76.2 \%$ LA yield was obtained after reaction for $12 \mathrm{~h}$.

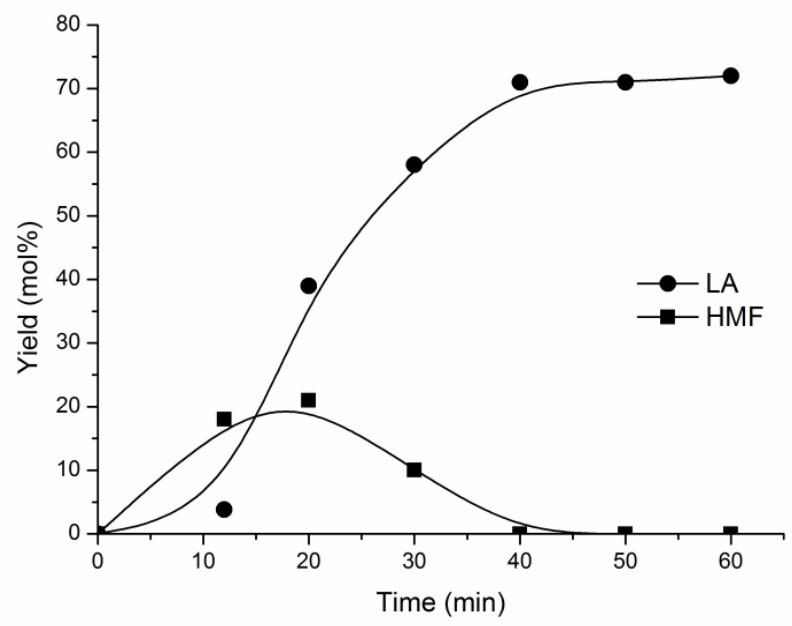

Figure 1. The yield of LA and HMF during fructose conversion Conditions: $0.925 \mathrm{mmol}$ fructose, $1.000 \mathrm{~g}\left[\mathrm{C}_{3} \mathrm{SO}_{3} \mathrm{Hmim}\right] \mathrm{HSO}_{4}, 6.000 \mathrm{~g}$ $\mathrm{H}_{2} \mathrm{O}, 170{ }^{\circ} \mathrm{C}$

The humin byproducts formed during fructose conversion were investigated by IR spectra (Fig. 2). After reaction for $12 \mathrm{~min}$, three bands at 1510, 1026 and $804 \mathrm{~cm}^{-1}$ were clearly observed, which are characteristic of furan rings vibrations and attributed to $\mathrm{C}=\mathrm{C}$ stretching vibrations, $\mathrm{C}-\mathrm{O}$ vibrations of furan rings and aromatic $\mathrm{C}-\mathrm{H}$ out-of-plane bending vibrations, respectively, indicating HMF could be linked into humin structure through aldehyde group predominantly [6]. Meanwhile, another set of two bands at 1674 and $1591 \mathrm{~cm}^{-1}$ were noticeable, which belong to $\mathrm{C}=\mathrm{O}$ and $\mathrm{C}=\mathrm{C}$ stretching vibrations in $\mathrm{HMF}$, respectively, illustrating the co-existence of 2-formylfuran ring linked into humin structure assumably via intermolecular dehydration of $\mathrm{O}-\mathrm{H}$ groups instead of aldehyde group in HMF [5]. Other characteristic bands observed in fructose humins are bands at ca. $3300 \mathrm{~cm}^{-1}$ attributed to $\mathrm{O}-\mathrm{H}$ stretching vibration, $3120 \mathrm{~cm}^{-1}$ assigned to aromatic C-H stretching vibrations, $2925 \mathrm{~cm}^{-1}$ assigned to aliphatic C-H stretching vibration, and $1705 \mathrm{~cm}^{-1}$ assigned to $\mathrm{C}=\mathrm{O}$ stretching vibrations conjugated with $\mathrm{C}=\mathrm{C}[10]$. At the early stage, HMF intermediate from fructose dehydration exists abundantly in the reaction system, therefore HMF can take part in humin formation through two reactive sites, including aldehyde group predominantly and hydroxyl group.

With the rise of reaction time from 12 to $50 \mathrm{~min}$, HMF intermediate was completely converted (Fig. 1). Humins can react further on aldehyde groups, as evidenced by the disappearance of the band at $1674 \mathrm{~cm}^{-1}$ assigned to $\mathrm{C}=\mathrm{O}$ stretching vibrations in 2-formylfuran rings. Consequently, the band of $\mathrm{C}=\mathrm{C}$ shifted from 1591 to $1616 \mathrm{~cm}^{-1}$, and the band of $\mathrm{O}-\mathrm{H}$ stretching vibrations moved to higher wavenumber of $3388 \mathrm{~cm}^{-1}$, presumably resulting from aldol addition and condensation of aldehyde $\mathrm{C}=\mathrm{O}$ groups. The three bands at 1510, 1026 and $804 \mathrm{~cm}^{-1}$ ascribed to furan rings vibrations continued to increase and dominated as polyfuranic units incorporated into humin structure. At this stage, when HMF intermediate was completely converted, humins can react further on aldehyde groups, as evidenced by disappearance of 2-formylfuran rings.

When the reaction was prolonged till $12 \mathrm{~h}$, three bands of furan rings at 1510, 1026 and $804 \mathrm{~cm}^{-1}$ as well as the band of aromatic C-H stretching vibrations at $3120 \mathrm{~cm}^{-1}$ decreased and became obscure, whereas the band of $\mathrm{C}=\mathrm{C}$ at $1616 \mathrm{~cm}^{-1}$ visibly increased, suggesting the opening of furan rings and dehydrogenation of aromatic hydrogen accompanied by further aromatization. The increased aromaticity was confirmed by the decrease of $\mathrm{H} / \mathrm{C}$ ratio 
from 0.724 to 0.686 through elemental analyses of humins after reaction for $80 \mathrm{~min}$ and $12 \mathrm{~h}$, also in line with the literature report that humin polyfuranics reacted further through intramolecular condensation, dehydration and decarboxylation reactions to generate extensively aromatic networks [11]. At the later stage, if the reaction time was long enough, humins can undergo subsequent aromatization, with the furan rings reduced and the $\mathrm{C}=\mathrm{C}$ groups increased.

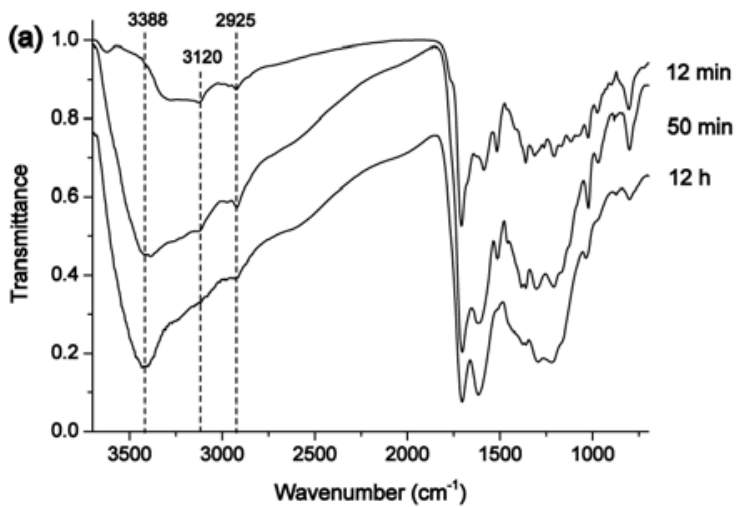

According to SEM images (Fig. 3), humins derived from fructose at early stages are mainly consisted of isolated and interconnected spherical particles. The average diameter of spherical particles grows from ca. $1 \mu \mathrm{m}$ (Fig. 3a-b) to $3 \mu \mathrm{m}$ (Fig. 3c-d) with the increase of reaction time from 12 to 50 min. After reaction for $12 \mathrm{~h}$, similar to $\mathrm{H}_{2} \mathrm{SO}_{4}$-catalyzed fructose conversion [6], agglomerated particles were observed resulting from further aromatization (Fig. 3e-f), which is in consistence with the IR analysis.

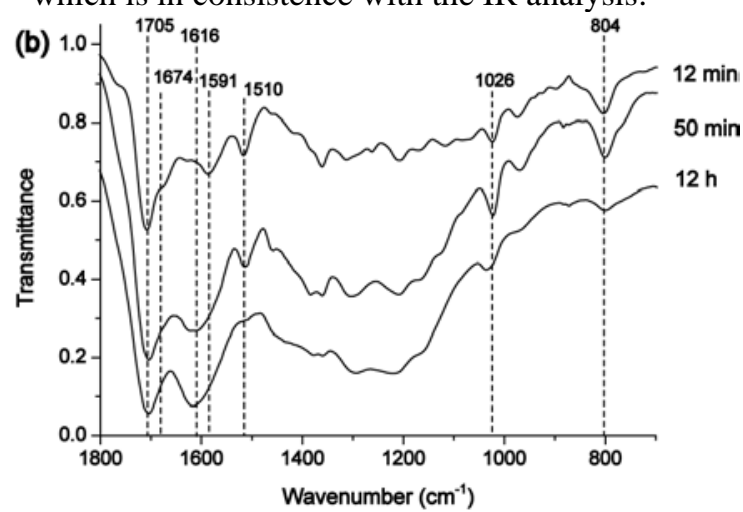

Figure 2. IR spectra of fructose humins: (a) $3700-700 \mathrm{~cm}^{-1}$; (b) $1800-700 \mathrm{~cm}^{-1}$. Conditions: $0.925 \mathrm{mmol}$ fructose, $1.000 \mathrm{~g}\left[\mathrm{C}_{3} \mathrm{SO}_{3} \mathrm{Hmim}^{\mathrm{HSO}} \mathrm{HS}_{4}, 6.000 \mathrm{~g}\right.$ $\mathrm{H}_{2} \mathrm{O}, 170{ }^{\circ} \mathrm{C}$
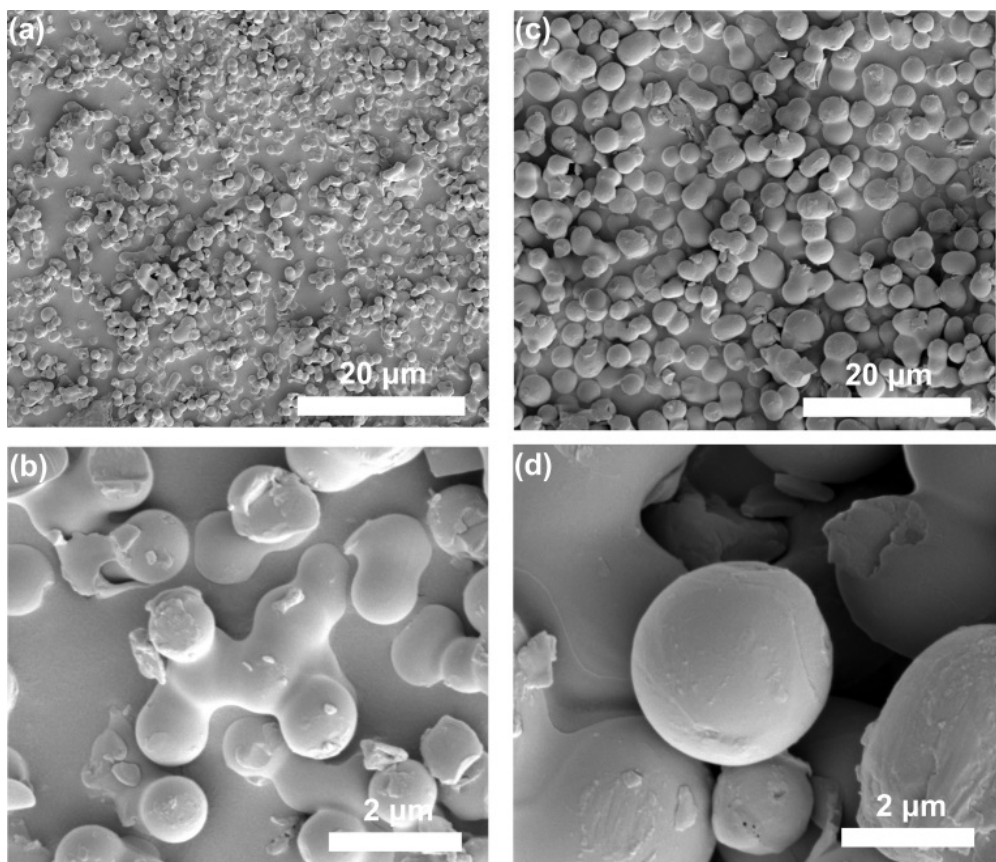
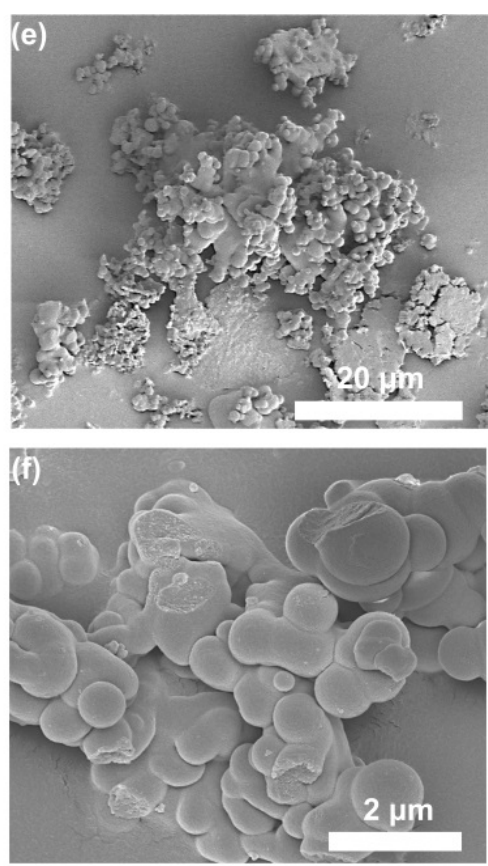

Figure 3. SEM images of humins formed during fructose conversion after reaction for (a-b) $12 \mathrm{~min}$, (c-d) $50 \mathrm{~min}$, (e-f) $12 \mathrm{~h}$. Conditions: $0.925 \mathrm{mmol}$ fructose, $1.000 \mathrm{~g}^{[}\left[\mathrm{C}_{3} \mathrm{SO}_{3} \mathrm{Hmim} \mathrm{HSO}_{4}, 6.000 \mathrm{~g} \mathrm{H}_{2} \mathrm{O}, 170{ }^{\circ} \mathrm{C}\right.$.

\section{CONCLUSIONS}

In summary, fructose was efficiently converted into LA with a high yield of $76 \%$ by catalysis of acidic ionic liquid in water. Through investigation on chemical structures and morphologies of humin byproduct, it was inferred that formation of fructose humins went through three stages for the first time. At the early stage, HMF intermediate from fructose dehydration can participate in humin structure through both aldehyde group predominantly and hydroxyl group. When HMF intermediate was completely converted, humins can undergo further reactions on aldehyde groups, with disappearance of 2-formylfuran rings. If the reaction time was long enough, humins can aromatize subsequently 
by reducing the furan rings and increasing the amount of $\mathrm{C}=\mathrm{C}$.

\section{ACKNOWLEDGMENT}

This project was supported by the Fundamental Research Funds for the Central Universities (DUT15LK17).

\section{REFERENCES}

[1] A. J. Ragauskas, C. K. Williams, B. H. Davison, G. Britovsek, J. Cairney, C. A. Eckert, W. J. Frederick, Jr., J. P. Hallett, D. J. Leak, C. L. Liotta, J. R. Mielenz, R. Murphy, R. Templer and T. Tschaplinski. "The path forward for biofuels and biomaterials," Science 2006, vol. 311, pp. 484-489.

[2] D. W. Rackemann and W. O. S. Doherty. "The conversion of lignocellulosics to levulinic acid," Biofuels Bioproducts \& Biorefining-Biofpr 2011, vol. 5, pp. 198-214.

[3] Y. Shen, Y. F. Xu, J. K. Sun, B. Wang, F. Xu and R. C. Sun. "Efficient conversion of monosaccharides into 5hydroxymethylfurfural and levulinic acid in $\mathrm{InCl}_{3}-\mathrm{H}_{2} \mathrm{O}$ medium," Catalysis Communications 2014, vol. 50, pp. 17-20.

[4] L. Qi, Y. F. Mui, S. W. Lo, M. Y. Lui, G. R. Akien and I. T. Horvath. "Catalytic conversion of fructose, glucose, and sucrose to 5(hydroxymethyl)furfural and levulinic and formic acids in gammavalerolactone as a green solvent," Acs Catalysis 2014, vol. 4, pp. 1470-1477.
[5] C. Yao, Y. Shin, L. Q. Wang, C. F. Windisch, W. D. Samuels, B. W Arey, C. Wang, W. M. Risen and G. J. Exarhos. "Hydrothermal dehydration of aqueous fructose solutions in a closed system," Journal of Physical Chemistry C 2007, vol. 111, pp. 15141-15145.

[6] S. K. R. Patil, J. Heltzel and C. R. F. Lund. "Comparison of structural features of humins formed catalytically from glucose, fructose, and 5hydroxymethylfurfuraldehyde," Energy \& Fuels 2012, vol. 26, pp. 5281-5293.

[7] I. van Zandvoort, Y. H. Wang, C. B. Rasrendra, E. R. H. van Eck, P. C. A. Bruijnincx, H. J. Heeres and B. M. Weckhuysen. "Formation, molecular structure, and morphology of humins in biomass conversion: influence of feedstock and processing Conditions," Chemsuschem 2013, vol. 6, pp. 1745-1758.

[8] H. Ren, Y. Zhou and L. Liu. "Selective conversion of cellulose to levulinic acid via microwave-assisted synthesis in ionic liquids," Bioresource Technology 2013, vol. 129, pp. 616-619.

[9] H. Ren, B. Girisuta, Y. Zhou and L. Liu. "Selective and recyclable depolymerization of celllose to levulinic acid catalyzed by acidic ionic liquid," Carbohydrate Polymers 2015, vol. 117, pp. 569-576.

[10] M. Sevilla and A. B. Fuertes. "The production of carbon materials by hydrothermal carbonization of cellulose," Carbon 2009, vol. 47, pp. 2281-2289.

[11] C. Falco, N. Baccile and M. M. Titirici. "Morphological and structural differences between glucose, cellulose and lignocellulosic biomass derived hydrothermal carbons," Green Chemistry 2011, vol. 13, pp. 3273-3281. 